\title{
Bose-Einstein condensation and superfluidity of dilute Bose gas in a random potential
}

\author{
Michikazu Kobayashi and Makoto Tsubota \\ Department of Physics, Osaka City University, Sumiyoshi-Ku, Osaka 558-8585, Japan
}

(Dated: October 31, 2018)

\begin{abstract}
There is a growing interest in the relation between Bose-Einstein condensation (BEC) and superfluidity. A Bose system confined in random media such as porous glass is suitable for studying this relation because BEC and superfluidity can be suppressed and controlled in such a disordered environment. However, it is not clear how this relation is affected by disorder and there are few theoretical studies that can be quantitatively tested by experiment. In this work, we develop the dilute Bose gas model with a random potential that takes into account the pore size dependence of porous glass. Then we compare our model with the measured low-temperature specific heat, condensate density, and the superfluid density of ${ }^{4} \mathrm{He}$ in Vycor glass. This comparison uses no free parameters. We predict phenomena at low temperatures that have not yet been observed. First, the random potential causes a $T$-linear specific heat instead of the $T^{3}$ dependence that is usually caused by phonons. Second, the BEC can remain even when the superfluidity disappears at low densities. And third, the system makes a reentrant transition at low densities; that is, the superfluid phase changes to the normal phase again as the temperature is reduced. This reentrant transition is more likely to be observed when the strength of the random potential is increased.
\end{abstract}

PACS numbers:

\section{INTRODUCTION}

Bose-Einstein condensation (BEC) and superfluidity of liquid ${ }^{4} \mathrm{He}$ in random environments including Aerogel and Vycor glass are active problems in quantum fluid research. In particular, finding out how spatial confinement affects the Bose fluid has stimulated both experimental and theoretical studies.

Below the $\lambda$ temperature of $2.17 \mathrm{~K}$, liquid ${ }^{4} \mathrm{He}$ enters the superfluid state and behaves as though it has no viscosity. Superfluidity is a macroscopic quantum phenomenon as well as superconductivity, and understanding both have been one of the major goals of quantum statistical physics. The various observations of superfluidity was successfully explained by the phenomenological two fluid model [1], which is based on the idea that the system consists of an inviscid superfluid and a viscous normal fluid. On the other hand, the lambda transition had been thought to be caused by BEC, which was confirmed by neutron scattering experiments [2. With a BEC, a macroscopic number of particles occupies a single particle ground state and is described by a macroscopic wave function. The inviscid superflow can be described by this wave function [3]. However the relation between BEC and superfluidity is not completely understood. Although superfluidity and BEC are closely related to each other, one is not necessary or sufficient for the other. For example, in a two-dimensional Bose system, Kosterlitz and Thouless proved that superfluidity can exist even without BEC [A], and the superfluidity was actually observed in ${ }^{4} \mathrm{He}$ films 55. The Bose system in a random environment might be another good example for studying the relation between BEC and superfluidity 6, 7. This system has received considerable attention because localization effects allow condensed particles to belong to the normal fluid rather than the superfluid and BEC can separate from the superfluid. The phase diagram of this system has been discussed, showing a specific nonsuperfluid phase. Thus, studies of this system can reveal the relation between BEC and superfluidity.

Porous glass such as Vycor is often used as a random media in experimental studies. Vycor glass is a from $30 \%$ to $70 \%$ porous glass containing wormholelike pores, the characteristic diameters of which vary from $30 \AA$ to $100 \AA$. By adjusting the pore size and the adsorbed ${ }^{4} \mathrm{He}$ coverage, we can change the density of ${ }^{4} \mathrm{He}$ and the superfluid transition. By using torsional oscillators, Reppy et al. 8, 9] interesting features at various pore sizes of Vycor glass or coverages were observed, particularly the behavior of the superfluid critical temperature and the temperature dependence of the superfluid density. The superfluid component has two-dimensional behavior when the pore size of Vycor glass is large, and becomes threedimensional as the pore size is reduced. The superfluid density in such porous glass is smaller than that of bulk ${ }^{4} \mathrm{He}$ and its critical temperature decreases with the coverage. Below a certain coverage, the superfluid density can no longer exist, even near $0 \mathrm{~K}$. These results show that superfluidity is broken by the random environment. It is also important to find out how disorder affects the BEC. BEC and its elementary excitation in liquid ${ }^{4} \mathrm{He}$ can be observed by neutron scattering. Bulk ${ }^{4} \mathrm{He}$ has the usual excitations such as phonons, maxons and rotons 1 . They are collective excitations on BEC, the excitations except for phonons being absent above the critical temperature. Dimeo et al. 10] and Plantevin et al. [11] used neutron scattering and a torsional oscillator to measure the elementary excitations and the superfluid transition, respectively, of ${ }^{4} \mathrm{He}$ in porous glass. Surprisingly, the dispersion curve in porous glass was the same as that in the bulk, which means that the disorder does not affect the elementary excitations. Furthermore, these elementary excitations were observed even above the superfluid crit- 
ical temperature. Hence, the BEC might persist in the disordered system even above the superfluid critical temperature. These results have not been understood completely yet; thus, the exact relationship between BEC and superfluidity remains puzzling.

This problem is also interesting from the following theoretical standpoint. In a Bose system confined in a random media, the long-range-order correlation due to BEC can compete with the disorder, so that the BEC critical temperature can be reduced. Huang and Meng proposed a model of the three-dimensional dilute Bose gas in a random potential [13] that assumed a small coverage of ${ }^{4} \mathrm{He}$ in Vycor glass . Because it is difficult to formulate the random potential for the porous glass, they used a delta-functional impurity potential and analyzed their model using the Bogoliubov transformation and taking an ensemble average. They found that both BEC and superfluidity are depressed by the random potential and the superfluidity disappears below a critical density, even at $0 \mathrm{~K}$, which is qualitatively consistent with the observations by Reppy et al. They also predicted a reentrant transition at low densities; that is, the superfluid phase enters a normal phase again with decreasing temperatures. However, the random potential of their model does not include the pore size, and thus it is difficult to quantitatively compare to experimental results for a range of pore sizes. Another model is the Bose Hubbard model with the random potential. By considering the transfer energy, the on-site repulsion, and the random potential, Fisher et al. 114 found that the Bose glass phase can exist with the superfluid phase and the Mott insulating phase. The Bose glass phase is similar to the Anderson insulating phase 15$]$ in metal. In the Bose glass phase, the condensed particles are localized and thus do not contribute to superfluidity. Thus, the Bose glass phase could influence the collective excitations even above the superfluid critical temperature. However, the theoretical excitation energy [16] for the Bose glass phase disagrees with measurements by neutron scattering experiment [11], so it is not yet clear whether the Bose glass phase has actually been detected. Finally, it should be noted that Huang and Meng's model cannot describe the Bose glass phase because the ensemble average makes the system uniform.

Few theoretical studies of this random system are quantitatively compare to the experiment. Thus, in this work, we improve Huang and Meng's model [13] by adding the size dependence of the random potential instead of using their the delta-functional potentials. The strength of the random potential can be estimated by comparing calculated and experimental critical coverages below which the superfluid density disappears, even at 0 $\mathrm{K}$. As a result, our model has no free parameters and can be used for quantitative comparisons to experimental data. This enables us to determine whether or not our picture of the three-dimensional dilute Bose gas in random potential is applicable to a real system. Our formulation cannot address this question at high tempera- tures due to the high number of thermally excited quasi particles. As far as the condensate density is almost independent of temperature at low temperatures, however, our formulation works well, leading to the following results. (1) The specific heat agrees quantitatively with experimental data at low temperatures. (2) Because of the random potential, the specific heat is not proportional to $T^{3}$, as occurs for phonons, but to $T$. Furthermore, by obtaining the condensate density and the superfluid density, we found the following. (3) When the total density is sufficiently low, BEC can persist even when the superfluid density disappears below that critical coverage. (4) The random potential causes a reentrant transition of the superfluid phase. Finally, we show why decreasing the open pore density of the Vycor glass should allow the reentrant phase to be detected experimentally

A brief summary of our paper is as follows. In Sec. II, we describe our model of the dilute Bose gas in a random potential and derive the partition function. Section III tests our model by quantitatively comparing calculated to experimental specific heats. In Sec. IV, the BEC density and the superfluid density are obtained and their characteristics are discussed. Section V is discussion and conclusions.

\section{MODEL}

Superfluid ${ }^{4} \mathrm{He}$ adsorbed in Vycor glass can be modeled by a three-dimensional dilute Bose gas in a random external potential [13]. The grand canonical Hamiltonian is

$$
\begin{aligned}
\hat{H}-\mu \hat{N} \equiv & \hat{K} \\
= & \int d x^{3} \hat{\Psi}^{\dagger}(\boldsymbol{x})\left[-\frac{\hbar^{2}}{2 m} \nabla^{2}+U(\boldsymbol{x})-\mu\right] \hat{\Psi}(\boldsymbol{x}) \\
& +\frac{v_{0}}{2} \int d x^{3} \hat{\Psi}^{\dagger}(\boldsymbol{x}) \hat{\Psi}^{\dagger}(\boldsymbol{x}) \hat{\Psi}(\boldsymbol{x}) \hat{\Psi}(\boldsymbol{x})
\end{aligned}
$$

where $\hat{\Psi}(\boldsymbol{x})$ is the field operator for Bose particles of mass $m, \hat{N}=\int d x^{3} \hat{\Psi}^{\dagger}(\boldsymbol{x}) \hat{\Psi}(\boldsymbol{x})$ is the number operator, $\mu$ is the chemical potential, and $U(\boldsymbol{x})$ is the external random potential that represents the effect of Vycor glass. The first term of the Hamiltonian is the kinetic energy and the external potential, whereas the second term refers to the hard-sphere interaction between particles with $v_{0}=4 \pi a \hbar^{2} / \mathrm{m}$ being the coupling constant with the s-wave scattering length $a$. This repulsive interaction prevents all particles from being localized at the minimum of $U(\boldsymbol{x})$. This has similarities to the Fermi system with disorder 15, 17, 18]; for example, Fermions cannot localize in a single orbital in space due to the Pauli exclusion principle. Therefore, the Fermion system is stable even if it is free from the repulsive interaction. On the other hand, to prevent the system from collapsing into the minimum of $U(\boldsymbol{x})$, the Bose system should include a repulsive interaction. This makes the problem more complicated than that of the Fermi system. 
Proceeding in a standard fashion, we introduce the free particle annihilation and creation operators $\hat{a}_{\boldsymbol{k}}$ and $\hat{a}_{\boldsymbol{k}}^{\dagger}$. We assume that the level with $\boldsymbol{k}=\mathbf{0}$ is macroscopically occupied with occupation number $N_{0}$, so $\hat{a}_{0}$ and $\hat{a}_{0}^{\dagger}$ are replaced by a c-number $\sqrt{N_{0}}$. By making a Fourier transformation and neglecting all off-diagonal terms $U_{\boldsymbol{k}} \hat{a}^{\dagger} \boldsymbol{k}^{\prime} \hat{a}_{\boldsymbol{k}^{\prime \prime}}$ and $v_{0} \hat{a}_{\boldsymbol{k}}^{\dagger} \hat{a}_{\boldsymbol{k}^{\prime}}^{\dagger} \hat{a}_{\boldsymbol{k}^{\prime \prime}} \hat{a}_{\boldsymbol{k}^{\prime \prime \prime}}$, we obtain

$$
\begin{aligned}
& \hat{K}_{\mathrm{eff}}=V\left(-\mu n_{0}+\frac{1}{2} v_{0} n_{0}^{2}+\frac{n}{V} U_{0}\right)+\sum_{\boldsymbol{k} \neq 0}\left[\frac{\hbar^{2} k^{2}}{2 m}-\mu+v_{0}\left(n+n_{0}\right)\right] \hat{a}_{\boldsymbol{k}}^{\dagger} \hat{a}_{\boldsymbol{k}}+\sqrt{\frac{n_{0}}{V}} \sum_{\boldsymbol{k} \neq 0}\left(U_{\boldsymbol{k}} \hat{a}_{\boldsymbol{k}}^{\dagger}+U_{-\boldsymbol{k}^{\hat{a}}}\right) \\
& +\frac{1}{2} v_{0} n_{0} \sum_{\boldsymbol{k} \neq 0}\left(\hat{a}_{\boldsymbol{k}}^{\dagger} \hat{a}_{-\boldsymbol{k}}^{\dagger}+\hat{a}_{\boldsymbol{k}} \hat{a}_{-\boldsymbol{k}}\right)
\end{aligned}
$$

where $V$ is the volume of the system, $n_{0}=N_{0} / V$ is the number density of condensate, and $U_{\boldsymbol{k}}$ is the Fourier transformation of $U(\boldsymbol{x})$. By neglecting the off diagonal terms, we are neglecting the interactions between the excited particle and the random potential and that between pairs of excited particles; these become important as the temperature rises and the condensate density decreases. Hence, this approximation is poor when many particles are thermally excited. Nevertheless, this approximation is useful at low temperatures where the condensate density is almost independent of temperature. All results here are obtained for these low temperatures.

This Hamiltonian can be diagonalized by the Bogoliubov transformation

$\hat{a}_{\boldsymbol{k}}=\frac{\hat{c}_{\boldsymbol{k}}+\gamma_{\boldsymbol{k}} \hat{c}^{\dagger}{ }_{\boldsymbol{k}}}{\sqrt{1-\gamma_{\boldsymbol{k}}^{2}}}+g_{\boldsymbol{k}}$

Then the coefficients $\gamma_{\boldsymbol{k}}, g_{\boldsymbol{k}}$ and the quasiparticle spectrum $\omega_{\boldsymbol{k}}$ are given by

$$
\begin{aligned}
\gamma_{\boldsymbol{k}} & =-\xi-1+\sqrt{\xi(\xi+2)} \\
g_{\boldsymbol{k}} & =-\sqrt{\frac{n_{0}}{V}} \frac{U_{\boldsymbol{k}}}{(\xi+2) v_{0} n_{0}}, \\
\omega_{\boldsymbol{k}} & =v_{0} n_{0} \sqrt{\xi(\xi+2)}, \\
\xi & =\frac{\hbar^{2} k^{2}}{2 m v_{0} n_{0}}+\Delta, \quad \Delta=\frac{v_{0} n_{0}-\mu}{v_{0} n_{0}} .
\end{aligned}
$$

Next, we take an ensemble-average to quench the random potential. The random potential simulates Vycor glass with a characteristic pore size $r_{p}$ as follows. The quenched potential $U_{\boldsymbol{k}}$ may decay above the characteristic wave number $k_{p}=2 \pi / r_{p}$. Thus, we assume the averaged potential

$\frac{1}{V}\left\langle U_{\boldsymbol{k}} U_{-\boldsymbol{k}}\right\rangle_{\mathrm{av}}=R_{0} \exp \left[-\frac{k^{2}}{2 k_{p}^{2}}\right]$,

where av denotes the ensemble-average. $R_{0}$, with dimension $(\text { energy })^{2}(\text { length })^{3}$, is the characteristic strength of the random potential. Equation (5) makes our model completely different from Huang and Meng's, and we will show that the results are also different. The coherence length of the BEC is thought to be from hundreds to thousands of $\AA$, whereas the spatial scale of disorder is the pore size in the glass, which is dozens of $\AA$. Hence, the macroscopic wave function of BEC is not sensitive to disorder in and between pores but instead depend on the disorder averaged over the coherence length. Hence, the ensemble-averaged system can become nearly uniform. For a uniform Bose system, it has been proven that the elementary excitation spectrum becomes the gapless Goldstone mode 19. Thus, we set $\Delta=0$ in Eq. (4d).

The resultant diagonalized and ensemble-averaged Hamiltonian is

$$
\begin{aligned}
\hat{K}_{\mathrm{eff}}= & V\left(-\mu n_{0}+\frac{n}{V} U_{0}+\epsilon_{1}+\epsilon_{R}\right) \\
& +\sum_{\boldsymbol{k} \neq 0} \hbar \omega_{k} \hat{c}_{\boldsymbol{k}}^{\dagger} \hat{c}_{\boldsymbol{k}}, \\
\omega_{k}= & \frac{\hbar}{2 m} k \sqrt{k^{2}+16 \pi a n_{0}}, \\
\epsilon_{1}= & \frac{2 \pi a n_{0}^{2} \hbar^{2}}{m}\left[1+\frac{128}{15 \sqrt{\pi}} \sqrt{n_{0} a^{3}}\right], \\
\epsilon_{R}= & \frac{m \sqrt{a} n_{0}^{3 / 2} R_{0}}{4 \sqrt{\pi} \hbar^{2}}\left[-\mathrm{e}^{2 \alpha}(5+4 \alpha)\{1-\operatorname{erf}(\sqrt{2 \alpha}\}\right. \\
\alpha= & \frac{4 \pi a n_{0}}{k_{p}^{2}},
\end{aligned}
$$

where $\epsilon_{1}$ is the hard sphere interaction energy at $0 \mathrm{~K}$, similarly, $\epsilon_{R}$ is that for the random potential. The quasiparticle spectrum $\omega_{k}$ is the same as that in the hard sphere Bose gas model [7] and is independent of the random potential. This independence is confirmed by neutron scattering experiments [1], which justifies the above assumption of $\Delta=0$; conversely, if $\Delta \neq 0$, the spectrum would depend on the random potential.

This Hamiltonian enables us to obtain the grand partition function $Q=\operatorname{Tr}\{\exp (-\beta \hat{K})\}$ and various physical 
quantities. The condensate density is defined by the following relation:

$n_{0}=n-\frac{1}{V} \sum_{\boldsymbol{k} \neq 0}\left\langle\hat{a}_{\boldsymbol{k}}^{\dagger} \hat{a}_{\boldsymbol{k}}\right\rangle$,

where $n$ is the particle number density. The second term represents the noncondensate particle number as

$$
\begin{aligned}
\frac{1}{V} \sum_{\boldsymbol{k} \neq 0}\left\langle\hat{a}_{\boldsymbol{k}}^{\dagger} \hat{a}_{\boldsymbol{k}}\right\rangle=n_{1}+n_{R}, & \\
n_{1}= & \frac{8}{3 \sqrt{\pi}}\left(n_{0} a\right)^{3 / 2} \\
& \quad+\frac{4}{\sqrt{\pi} \lambda^{3}} \int_{0}^{\infty} d t \frac{t\left(t^{2}+\theta / 2\right)}{\sqrt{t^{2}+\theta}\left\{\mathrm{e}^{t \sqrt{t^{2}+\theta}}-1\right\}}, \\
n_{R}= & \frac{m^{2} R_{0}}{8 \pi^{3 / 2} \hbar^{4}} \sqrt{\frac{n_{0}}{a}}\left[\mathrm{e}^{2 \alpha}(1+4 \alpha)\{1-\operatorname{erf}(\sqrt{2 \alpha})\}\right. \\
\lambda= & \sqrt{\frac{2 \pi \beta \hbar^{2}}{m}}, \quad \theta=\frac{\left.-2 \sqrt{\frac{2 \alpha}{\pi}}\right],}{m}, \quad t^{2}=\frac{\hbar^{2} \beta}{2 m} k^{2} .
\end{aligned}
$$

Here $n_{1}$ is the noncondensate density excited by the hard sphere interaction, $n_{R}$ is the density due to the scattering of condensate particles with the random potential, and $\lambda$ is the thermal de Broglie wave length. When $a$ vanishes, $n_{R}$ becomes infinite. This means that the system would collapse if there were no repulsive interactions between particles.

Because superfluidity is described by the two fluid model, the particle density $n$ consists of the normal fluid density $n_{n}$ and the superfluid density $n_{s}$. The superfluid density $n_{s}$ can be calculated by linear response theory [20. Because of its viscosity, only the normal fluid responds to a small, applied velocity field. Thus the normal fluid density can be defined by the response of the momentum density $j_{i}(\boldsymbol{x}, t)$ to the external velocity field $v_{i}(\boldsymbol{x}, t)$. Linear response theory gives the following relation:

$$
\begin{aligned}
j_{i}(\boldsymbol{x}, t)= & \chi_{i j}(\boldsymbol{x}, t) v_{j}(\boldsymbol{x}, t), \\
\chi_{i j}(\boldsymbol{x}, t)= & \left\langle\left[j_{i}(\boldsymbol{x}, t), j_{j}(0,0)\right]\right\rangle \\
j_{i}(\boldsymbol{x}, t)= & \frac{\hbar}{2 i}\left\{\hat{\Psi}^{\dagger}(\boldsymbol{x}, t) \frac{\partial \hat{\Psi}(\boldsymbol{x}, t)}{\partial x_{i}}\right. \\
& \left.\quad-\frac{\partial \hat{\Psi}^{\dagger}(\boldsymbol{x}, t)}{\partial x_{i}} \hat{\Psi}(\boldsymbol{x}, t)\right\} \\
\hat{\Psi}(\boldsymbol{x}, t)= & \mathrm{e}^{\mathrm{i}(\hat{H}-\mu \hat{N}) t / \hbar} \hat{\Psi}(\boldsymbol{x}) \mathrm{e}^{-\mathrm{i}(\hat{H}-\mu \hat{N}) t / \hbar},
\end{aligned}
$$

where $\hat{\Psi}(\boldsymbol{x}, t)$ is the Heisenberg field operator. The static susceptibility $\chi_{i j}(\boldsymbol{k})$ is defined as

$$
\begin{aligned}
\chi_{i j}(\boldsymbol{x}, t) & =\int \frac{d \omega}{2 \pi} \frac{d^{3} k}{(2 \pi)^{3}} \mathrm{e}^{-\mathrm{i} \omega t} \mathrm{e}^{\mathrm{i} \boldsymbol{k} \cdot \boldsymbol{x}} \chi_{i j}(\boldsymbol{k}, \omega), \\
\chi_{i j}(\boldsymbol{k}) & =\lim _{\omega \rightarrow 0} \chi_{i j}(\boldsymbol{k}, \omega) .
\end{aligned}
$$

Because of the rotational invariance, the static susceptibility $\chi_{i j}(\boldsymbol{k})$ can be written

$\chi_{i j}(\boldsymbol{k})=\frac{k_{i} k_{j}}{k^{2}} A(\boldsymbol{k})+\left(\delta_{i j}-\frac{k_{i} k_{j}}{k^{2}}\right) B(\boldsymbol{k})$,

where $A(\boldsymbol{k})$ and $B(\boldsymbol{k})$ are the longitudinal and transverse parts, respectively. The transverse susceptibility $B(0)$ is the normal fluid mass density. The superfluid number density $n_{s}$ is $n-B(0) / m$. The susceptibility $B(0)$ can be calculated by the Bogoliubov transformation in Eq. (3). After some tedious calculations, the resultant superfluid density is given by

$$
\begin{aligned}
& n_{s}=n-n_{n 1}-n_{n R}, \\
& n_{n 1}=\frac{8}{3 \sqrt{\pi} \lambda^{3}} \int_{0}^{\infty} d t \frac{t^{4} \mathrm{e}^{-t \sqrt{t^{2}+\theta}}}{\left(1-\mathrm{e}^{-t \sqrt{t^{2}+\theta}}\right)^{2}} \\
& n_{n R}=\frac{4}{3} n_{R},
\end{aligned}
$$

where $n_{n 1}$ is the normal fluid density due to the elementary excitations, and $n_{n R}$ is that due to scattering with the random potential. The density $n_{n 1}$ can be also obtained using Khalatnikov's method that is based on Galilean invariance [21]. The relation $n_{n R}=4 / 3 n_{R}=$ $n_{R}+1 / 3 n_{R}$ shows that the random potential causes the larger normal fluid density than the noncondensate density; some condensate particles are captured by the random potential to participate in the normal fluid. This makes it possible to destroy superfluidity even at $0 \mathrm{~K}$ when $n_{n R}$ becomes comparable to $n$.

This formulation can be used to obtain various physical quantities including the condensate density, the superfluid density, and the specific heat.

\section{COMPARISON WITH EXPERIMENTS}

In this section, we compare the calculated specific heat and the superfluid density with experimental results. Quantitative agreement is shown to be good at low temperatures, which supports our assumption of a dilute Bose gas in the random potential. Furthermore, we show that the random potential leads to as-yet unobserved behavior of the specific heat.

To make a quantitative comparison, we give the following numerical values to the parameters: $m \simeq 6.6 \times$ $10^{-27} \mathrm{~kg}$ and $a \simeq 5 \times 10^{-10} \mathrm{~m}$ are the mass and the s-wave scattering length of a ${ }^{4} \mathrm{He}$ atom. Other parameters are from the experiments of Reppy et al. [8]. The volume $V$ of open pores in the Vycor glass (about $40 \%$ of the total volume of the Vycor glass) is about $1 \mathrm{~cm}^{3}$. The particle density $n$ of ${ }^{4} \mathrm{He}$ inside the Vycor glass is estimated as follows. In Vycor glass, the atoms are adsorbed and fully cover the surfaces of the open pores due to the van der waals attraction. The pore area is about $10^{8} \mathrm{~m}^{2} / \mathrm{m}^{3}$. The rest of atoms, which do not participate in the firstlayer solid, can behave as a dilute gas inside the pores. The particle density $n$ of the dilute gas is obtained by 
subtracting the adsorbed amount from the total amount. This density is estimated to be from $0.001 \%$ to $70 \%$ of the density of bulk liquid ${ }^{4} \mathrm{He} n_{\text {bulk }} \sim 2.1 \times 10^{28} / \mathrm{m}^{3}$. Because the first layer of ${ }^{4} \mathrm{He}$ adsorbed on the surfaces cannot move and behaves as a solid, we assume the pore size $30 \AA$ of Vycor glass is effectively reduced by $2 a$. Thus, $r_{p}$ is estimated to be $20 \AA$. The last parameter $R_{0}$, which is the strength of the random potential, can be fixed by comparing to experiment. Shown in Fig. 11(a) are the data of zero temperature superfluid signals taken in an experiment that used a torsional oscillator (Fig. 12 of reference [8]). Because the superfluid component does not contribute to the moment of inertia, the resonant frequency and the period of oscillation differ from those without superfluid. The period difference $\Delta P$ is approximately proportional to the superfluid component. Here, the superfluid density is nearly proportional to $\Delta P$ and disappears at a coverage of $17.5 \mathrm{mg}$. Figure 1 (b) shows the superfluid density at $0 \mathrm{~K}$ from Eq. (12). As in the experiment, the superfluid density becomes zero at a certain coverage that depends on $R_{0}$. Thus, the value of $R_{0}$ can be fixed using the comparison with Fig. 11(a); i.e., $R_{0}=5 \times 10^{-75} \mathrm{~J}^{2} \mathrm{~m}^{3}$. Here, we define $R_{w} \equiv \sqrt{R_{0} n}$, which is the single particle energy converted from $R_{0}$. In the Vycor glass, $R_{w} / k_{B}$ is about $0.001 \sim 1 \mathrm{~K}$. Just above the critical coverage, the superfluid density increases linearly for both the experiment and the calculation; however, their slopes cannot be compared because the amplitude of $n_{s}$ is unknown in the experiment.

Because all parameters are now fixed, we will quantitatively compare calculations to experiments. The specific heat can be obtained from temperature differentiation of the free energy:

$$
\begin{aligned}
\Omega= & \Omega_{1}+\Omega_{R} \\
\Omega_{1}= & V\left(-\mu n_{0}+\epsilon_{1}\right) \\
& +\frac{4 V}{\sqrt{\pi} \beta \lambda^{3}} \int_{0}^{\infty} d t\left\{t^{2} \log \left[1-\mathrm{e}^{-t \sqrt{t^{2}+\theta}}\right]\right\}, \\
\Omega_{R}= & V\left(\epsilon_{R}+\frac{n}{V} U_{0}\right) .
\end{aligned}
$$

where $\Omega_{1}$ is the free energy of the elementary excitation and the hard sphere interaction, and $\Omega_{R}$ is the free energy from the random potential. Figure 2 compares our results to data of low temperature specific heat taken from Fig. 1 of reference [8]. In Fig. 2(a), which shows the data for high density, the density $n$ is fixed from the experimental coverage, whereas we fix the density from the superfluid critical temperature in Fig. 2(b) at low density. This is because we have no information about the data on the coverage. Figure 2(b) also shows the superfluid density. The theoretical results agree quantitatively with experiment without using free parameters. Above $1.0 \mathrm{~K}$ in Fig. 2(a), the calculated condensate density begins to decrease rapidly; here, our criterion of constant condensate density fails, which likely causes the discrepancy with experiment. However, Fig. 2(b) shows that the calculated specific heat agrees with experiment up to temperatures near the superfluid critical temperature; in

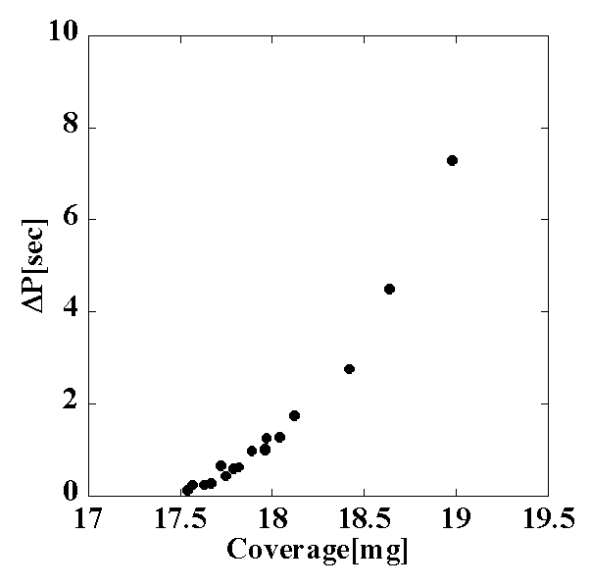

(a)

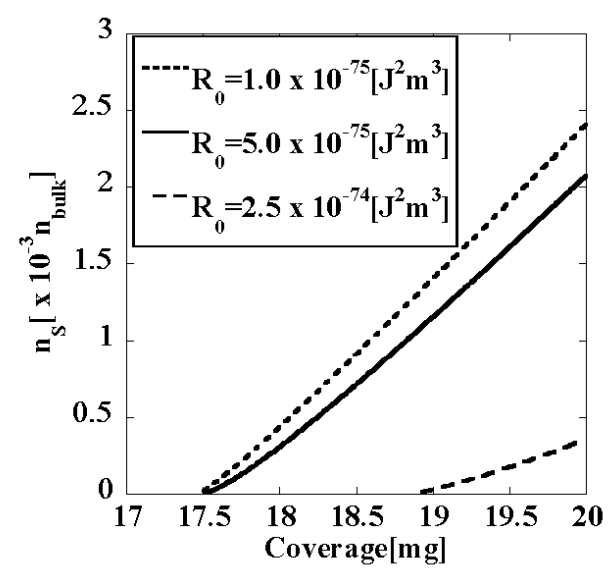

(b)

FIG. 1: Superfluid signals of experiments (a) and calculations (b) near $0 \mathrm{~K} . \Delta P$ is the resonant period difference in a torsional balance experiment, which is approximately proportional to the superfluid density [8].

this temperature region, the calculated condensate density hardly decreases. This means that the system is more dilute than that of Fig. 2(a) and thus is affected by the random potential rather than the elementary excitations. We discuss this in the next section. Nevertheless, these comparisons show that our model is accurate at low temperatures.

Our model predicts an effect at low temperature that is due to the random potential but has not yet been observed. This is shown in Fig. 3, which is the log-log plot of Fig. 2(a). When the system is free from the random potential, the specific heat should increase a $T^{3}$ because of the contribution from the phonons. However, with a random potential, the dependence is linear in $T$ at low temperatures. This means that at low temperatures, the 


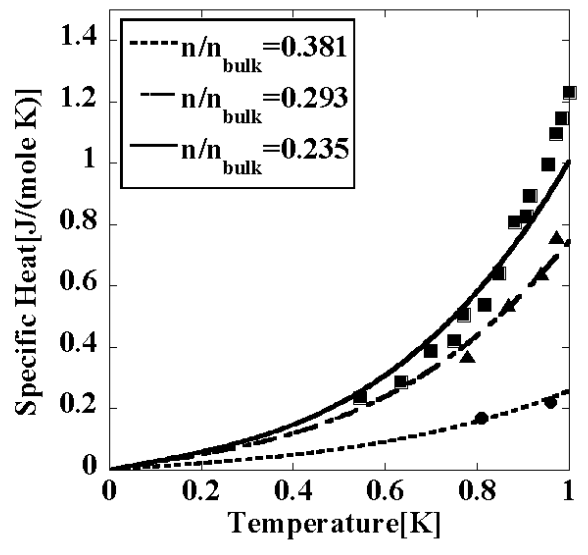

(a)

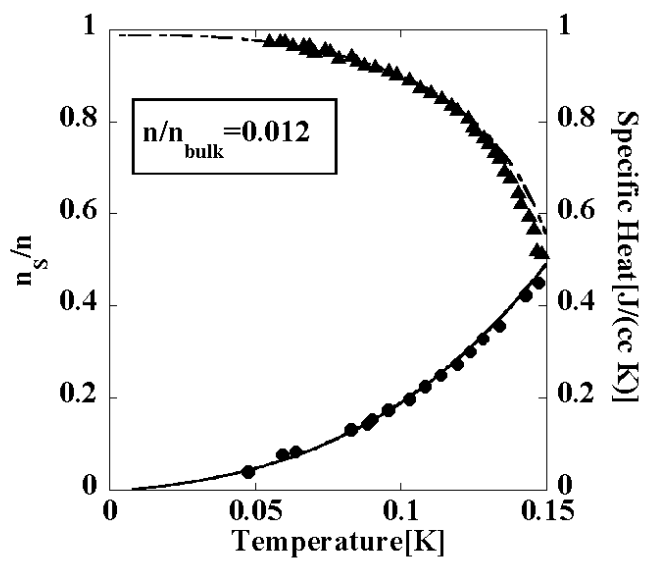

(b)

FIG. 2: The specific heat data from in experiments (plot) and calculations (line). In (a), experimental data are given by Fig.1 of reference [- The circles, triangles and squares respectively correspond to full pores $(\sigma=1), \sigma=0.780$ and $\sigma=0.636$. Here $\sigma$ is the ratio of the coverage to the full pore coverage. In (b), calculated and experimental superfluid densities are compared $\left(T_{c}=0.163 \mathrm{~K}\right)$.

contribution from the random potential is larger than that from the elementary excitations (phonons). The free energy $\Omega_{R}$ in Eq. (13) depends on the temperature only through the condensate density $n_{0} . \Omega_{R}$ is the energy from the scattering of the condensate particles with the random potential, and the resultant specific heat is given by the energy that the condensate particles need to slip out of the random potential. Experimental observation of this $T$-linear dependence might clearly identify the influence from the random potential.

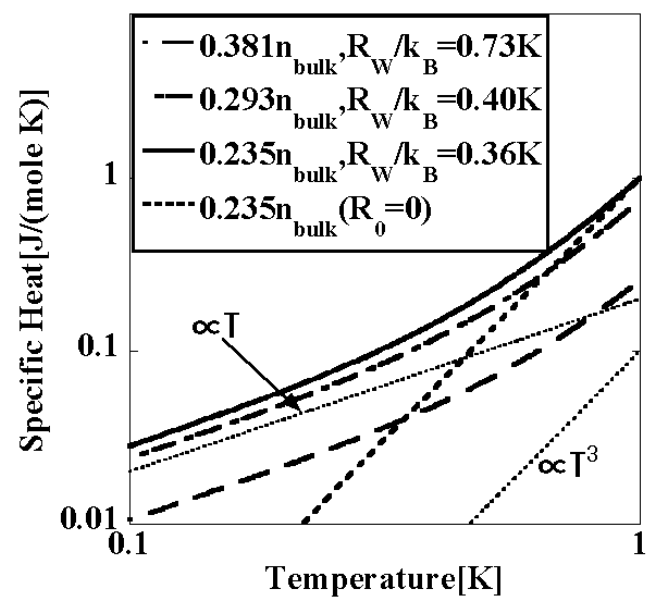

FIG. 3: The log-log plot of Fig. 2(a). Data of the specific heat at $R_{0}=0\left(n / n_{\text {bulk }}=0.35\right)$. Two lines for $\propto T$ and $\propto T^{3}$ are added.

\section{CONDENSATE DENSITY AND SUPERFLUID DENSITY}

This section describes some characteristic behavior of the condensate density $n_{0}$ and the superfluid density $n_{s}$ derived from our model.

Figure. 4 shows the dependence of $n_{0}$ and $n_{s}$ on temperature and density. Both $n_{0}$ and $n_{s}$ decrease with decreasing density, even at $0 \mathrm{~K}$. This means that the effect of the random potential on $n_{0}$ and $n_{s}$ becomes larger as the density is reduced. Figure. 1 shows clearly the difference between $n_{s}$ and $n_{0}$. Below the critical density, the superfluid density disappears, although the condensate persists. This situation indicates that the condensate particles cannot move as a superfluid because they are trapped by the random potential. We expect that this theoretical result will be confirmed by measurements of condensate density.

Figure. 5 shows the temperature dependence of the superfluid density just before superfluidity disappears. This figure shows the reentrant transition at which the superfluid density $n_{s}$ goes to zero with a decrease of temperature. In this temperature region, the condensate density is almost constant; hence, our formulation should work well in accordance with the criterion described in Sec. II. This reentrant transition is understood as follows. The condensate depletion $n_{R}$ of Eq. (88c) comes from the scattering of the condensate particles with the random potential. This decreases with $n_{0}$ as the temperature rises, so that $n_{n R}=4 / 3 n_{R}$ of Eq. (12k) also decreases. As shown in Fig. 6, the magnitude of this decrease in $n_{n R}$ exceeds the increase in $n_{n 1}$ of Eq. $(12 \mathrm{~b})$, which is the normal fluid density due to the elementary excita- 


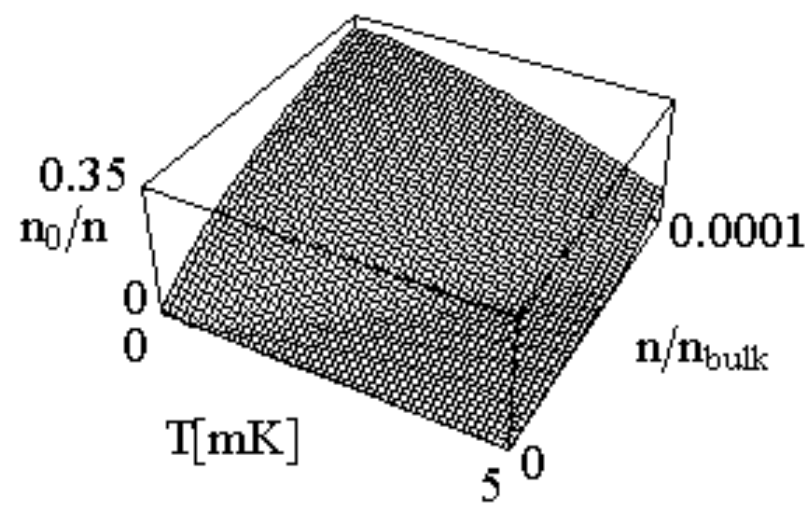

(a)

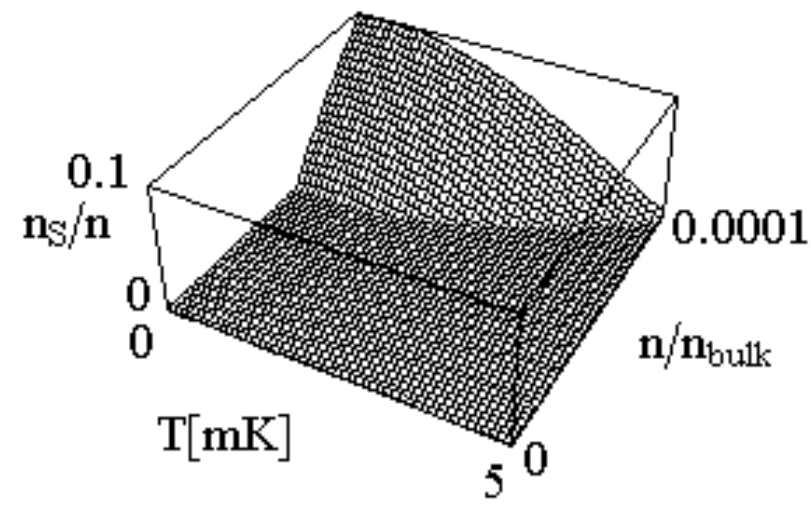

(b)

FIG. 4: Temperature and density dependence of $n_{0}$ (a) and $n_{s}$ (b) at $R_{0}=5.0 \times 10^{-75} \mathrm{~J}^{2} \mathrm{~m}^{3}$.

tions, in the very low temperature region $T \leq 0.5 \mathrm{mK}$. In other words, condensate particles that are trapped by the random potential at lower temperatures can escape at higher temperatures and thus participate in superfluidity. This reentrant transition has not been observed experimentally, probably because it should only occur at very low densities and low temperatures. However, large values of $R_{0}$ can make the reentrant transition observable as follows. We define the temperature $T_{\max }$ as that which maximizes the superfluid density $n_{s}$, and define $\Delta n_{s} \equiv n_{s}\left(T=T_{\max }\right)-n_{s}(T=0)$. Fig. 7 shows the density dependence of $T_{\max }$ and $\Delta n_{s}$. Both variables increase with $R_{0}$. Therefore, the reentrant transition is more likely to be measured at larger $R_{0}$. The parameter $R_{0}$ is the strength of the random potential over the entire space, and one way to increase $R_{0}$ is to decrease the open pore density of the Vycor glass.

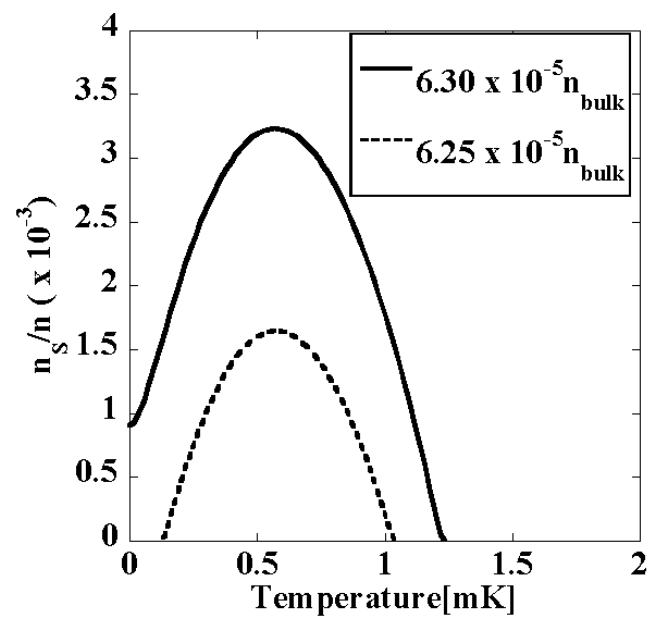

FIG. 5: Temperature dependence of $n_{s}$ at low temperature and low density. In this regime, the superfluid density $n_{s}$ goes to zero with a decrease of temperature (reentrant transition).

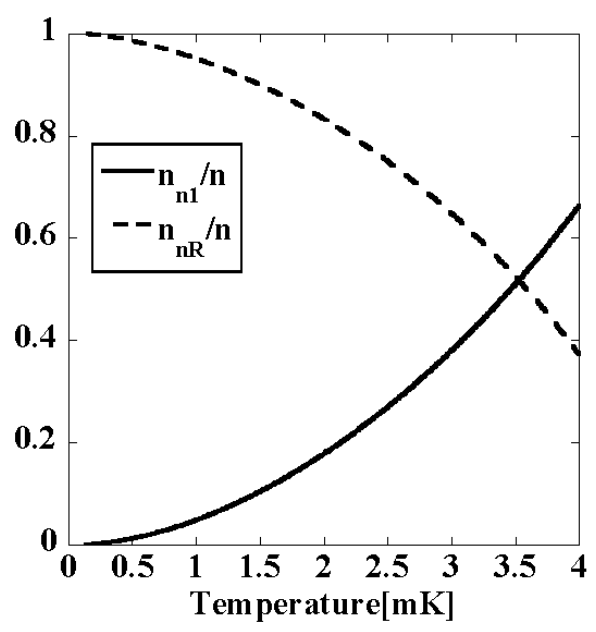

FIG. 6: Temperature dependence of $n_{n 1}$ and $n_{n R}$ near the reentrant distribution at $n=6.25 \times 10^{-5} n_{b u l k}$ (dashed line in Fig. 1).

\section{CONCLUSIONS}

The present paper describes the dilute Bose gas system in a random potential. The outcomes of our studies are as follows.

By including the pore size dependence of Vycor glass in the random potential, our model could closely match the experimental conditions of liquid ${ }^{4} \mathrm{He}$ in Vycor glass. We fixed the strength of the random potential by equating 


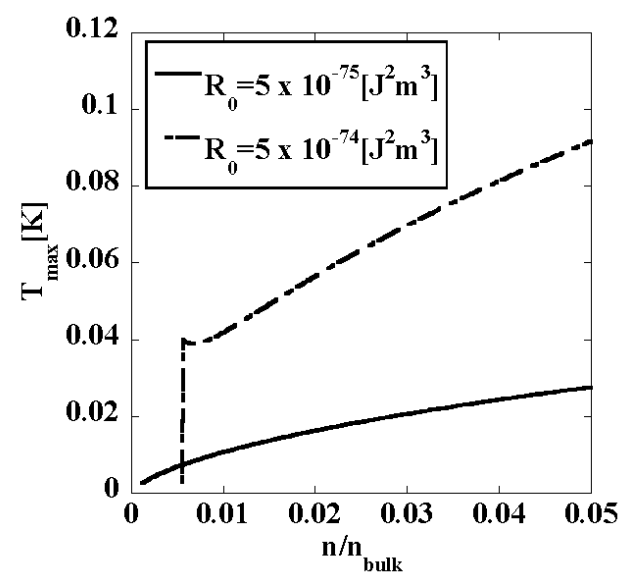

(a)

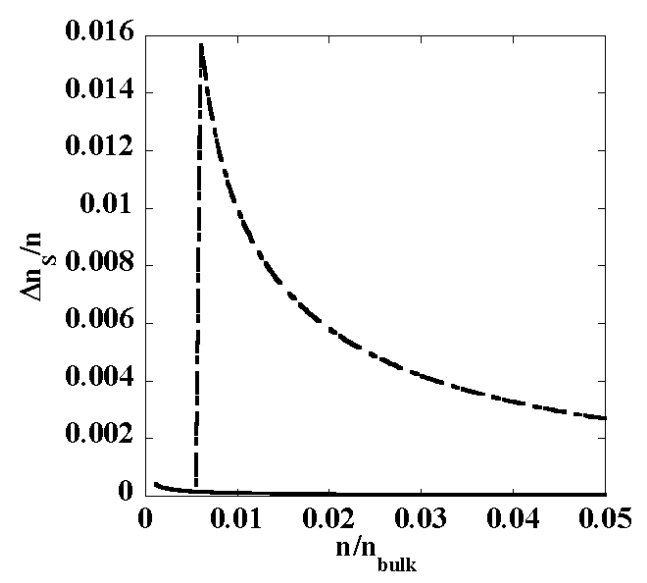

(b)

FIG. 7: Density dependence of $T_{\max }$ and $\Delta n_{s}$ for two values of $R_{0} . T_{\max }$ is the temperature that maximizes the superfluid density $n_{s}$, and $\Delta n_{s}=n_{s}\left(T=T_{\max }\right)-n_{s}(T=0)$. the theoretical and experimental critical coverages below which the superfluid density at $0 \mathrm{~K}$ vanishes. No other parameters could be adjusted, and thus we could quantitatively compare theory to experiment for other physical quantities.

First, we showed that the calculated specific heat for Vycor glass quantitatively agrees with measurements. This indicates that liquid ${ }^{4} \mathrm{He}$ in Vycor glass behaves as a dilute Bose gas in a random potential. For low temperatures, the calculated specific heat was linear in $T$ because of the random potential. Second, the BEC was shown to persist even when superfluidity disappears below the critical density. Finally, we showed that the reentrant transition of the superfluid phase is more likely to be observed experimentally by increasing the strength of the random potential.

Because we neglected interactions between pairs of excited particles and between excited particles and the random potential, this model does not apply to systems at high temperatures. To overcome this limitation, we are improving the model to include these interactions and will report on this more general model in the near future.

\section{Acknowledgments}

MT acknowledges support by a Grant-in-Aid for Scientific Research (Grant No.12640357) by Japan Society for the Promotion of Science.
[1] D.R.Tilley and J.Tilley, Superfluidity and Superconductivity (Institute of Physics Publishing Bristol and Philadelphia, 1990), Third Edition.

[2] P.E.Sokol, in Bose-Einstein Condensation (Cambridge University Press, 1995), edited by A.Griffin, D.W.Snoke and S.Stringari, p.51.

[3] K.Huang, Statistical Mechanics (John Wiley and Sons, 1987), Second Edition.

[4] J.M.Kosterlitz and D.J.Thouless, J.Phys. C6(1973)1181.

[5] D.J.Bishop and J.D.Reppy, Phys. Rev. Lett. 40(1978) 1727

[6] N.Trivedi, in Computer Simulation Studies in Condensed Matter Physics V, (Springer, Berlin, 1993); W.Krauth, N.Trivedi and D.Ceperley, Phys. Rev. Lett. 67(1991)2307.
[7] K.Huang, in Bose-Einstein Condensation (Cambridge University Press, 1995), edited by A.Griffin, D.W.Snoke and S.Stringari, p.31.

[8] J.D.Reppy, J. Low Temp. Phys. 87(1992)205.

[9] B.C.Crooker, B.Hebral, E.N.Smith, Y.Takano and J.D.Reppy, Phys. Rev. Lett. 51(1983)666.

[10] R.M.Dimeo, P.E.Sokol, C.R.Anderson, W.G.Stirling, K.H.Andersen and M.A.Adams, Phys. Rev. Lett. 81(1998)5860.

[11] O.Plantevin, B.Fåk, H.R.Glyde, N.Mulders, J.Bossy, G.Coddens and H.Schober, Phys. Rev. B63(2001)224508.

[12] C.J.Pethick and H.Smith, Bose-Einstein Condensation in Dilute Gases, (Camdridge University Press, 2002).

[13] K.Huang and H.F.Meng, Phys. Rev. Lett. 69(1992)644. 
[14] M.P.A.Fisher, P.B.Weichman, G.Grinstein and D.S.Fisher, Phys. Rev. B40(1989)546.

[15] P.W.Anderson, Phys. Rev. 109(1958)1492.

[16] W.Krauth, N.Trivedi and D.Ceperley, Phys. Rev. Lett. 67(1991)2307.

[17] J.S.Langer and T.Neal, Phys. Rev. Lett. 16(1966)984.

[18] L.P.Gor'kov, A.I.Larkin and D.E.Khmel'nitskii, JETP Lett. 30(1979) 248
[19] S.Stringari, in Bose-Einstein Condensation (Cambridge University Press, 1995), edited by A.Griffin, D.W.Snoke and S.Stringari, p.86.

[20] P.C.Hohenberg and P.C.Martin, Ann. Phys. (N.Y.) 34(1965)291.

[21] I.M.Khalatnikov, Introduction to the Theory of Superfluidity, (Benjamin, New York, 1965), p.13. 